\title{
Gastrointestinal involvement in COVID-19 patients: a retrospective study from a Greek COVID-19 referral hospital
}

\author{
Panagiotis Tsibouris ${ }^{a}$, Konstantinos Ekmektzoglou ${ }^{a, b}$, Alexandra Agorogiannic, \\ Chrysostomos Kalantzis ${ }^{a}$, Antonia Theofanopoulou ${ }^{a}$, Klearchos Toumbelis ${ }^{a}$, \\ Leonidas Petrogiannopoulos ${ }^{a}$, Charalambos Poutakidis a, Stavroula Goggakia, loannis Braimakisa, \\ Erasmia Vlachou ${ }^{\mathrm{a}}$, Abraham Pouliakis ${ }^{\mathrm{d}}$, Periklis Apostolopoulos ${ }^{\mathrm{a} *}$ \\ Army Share Fund Hospital (NIMTS), Athens, Greece; European University Cyprus, Nicosia, Cyprus; "ATTIKON" \\ University Hospital, National and Kapodistrian University of Athens, Athens, Greece
}

Abstract

\begin{abstract}
Background Much attention has been paid to the study and reporting of gastrointestinal (GI) symptoms in COVID-19 patients. Moreover, an increasing number of COVID-19 patients have been noted to experience hepatic and pancreatic injury. In this study, we retrospectively investigated symptoms and laboratory findings related to the GI system in a single center in Athens, Greece, and assessed the role of these parameters in relation to survival and disease severity.
\end{abstract}

Method We retrospectively studied 61 adult COVID-19 patients admitted to the Army Share Fund Hospital (NIMTS) in Athens, Greece, from April 6th to May 6 ${ }^{\text {th }}, 2020$.

Results Sixty-one COVID-19 cases were assessed in the study period. Regarding both survival and disease severity, diarrhea was the most common finding. The multivariate analysis revealed that elevated serum aspartate aminotransferase levels and low serum albumin levels were associated with worse patient survival (odds ratio [OR] 1.029, 95\% confidence interval [CI] 1.007-1.05, $\mathrm{P}=0.0088$; and $\mathrm{OR} 0.219,95 \% \mathrm{CI} 0.066-0.723, \mathrm{P}=0.0127$, respectively). As far as disease severity is concerned, only a low serum albumin level (measured at hospital admission) was correlated with more severe disease (OR 0.025, 95\% CI 0.004-0.161, $\mathrm{P}=0.0001$ ).

Conclusions Outpatients with new-onset GI symptoms should be considered for COVID-19 testing in a high COVID-19 prevalence setting, as these symptoms are observed more and more in clinical settings. As prospective studies begin to emerge, clinicians will have more robust research data to diagnose COVID-19 patients earlier and identify patients in need of more intensive treatment.

Keywords COVID-19, SARS-CoV-2, gastrointestinal symptoms, Greece, retrospective

Ann Gastroenterol 2020; 33 (5): 1-8

aDepartment of Gastroenterology, Army Share Fund Hospital (NIMTS), Athens, Greece (Panagiotis Tsibouris, Konstantinos Ekmektzoglou, Chrysostomos Kalantzis, Antonia Theofanopoulou, Klearchos Toumbelis, Leonidas Petrogiannopoulos, Charalambos Poutakidis, Stavroula Goggaki, Ioannis Braimakis, Erasmia

Vlachou, Periklis Apostolopoulos); 'bchool of Medicine, European University Cyprus, Nicosia, Cyprus (Konstantinos Ekmektzoglou); ${ }^{c} 2^{\text {nd }}$ Department of Internal Medicine, Army Share Fund Hospital (NIMTS), Athens, Greece (Alexandra Agorogianni); ${ }^{\mathrm{d}}{ }^{\text {nd }}$ Department of Pathology, "ATTIKON" University Hospital, National and Kapodistrian University of Athens, Athens, Greece (Abraham Pouliakis)

Conflict of Interest: None

Correspondence to: Periklis Apostolopoulos MD, $\mathrm{PhD}, 10-12$ Monis Petraki Street, 11523, Athens, Greece, e-mail: periklisapo@hotmail.com

Received 4 June 2020; accepted 8 June 2020;

published online 30 June 2020

DOI: https://doi.org/10.20524/aog.2020.0514

\section{Introduction}

In December 2019, pneumonia caused by a novel coronavirus, severe acute respiratory syndrome coronavirus 2 (SARS-CoV-2), broke out in Wuhan (Hubei province, China) and subsequently spread around the world [1,2]. Up to now, figures regarding coronavirus disease 2019 (COVID-19) are disheartening, as roughly 8 million cases have been reported worldwide, with more than 434,000 deaths being recorded $[3,4]$.

Much attention has been paid to the study and reporting of gastrointestinal (GI) manifestations, since SARS-CoV-2 ribonucleic acid (RNA) can be detected in stool specimens, with prolonged virus shedding being reported in patients with diarrhea [2,5-7]. The presence of GI symptoms is linked with a $70 \%$ increased risk of testing positive for SARS-CoV-2 [8]. 
Pooled prevalence estimates in hospitalized patients are $7.7 \%$ for diarrhea, $7.8 \%$ for nausea/vomiting and $2.7 \%$ for abdominal pain. Even though estimates are higher in studies outside of China, according to the latest American Gastroenterological Association (AGA) Recommendations for the Consultative Management of Patients with COVID-19, symptoms are associated with COVID-19 in less than $10 \%$ of patients [9]. However, GI symptoms do not seem to affect disease course [5].

Nevertheless, an increasing number of COVID-19 patients have been noted to experience hepatic injury, ranging over a spectrum from mild to severe damage [10]. To complicate matters even more, a recent study reported pancreatic injury in COVID-19 patients (defined by any abnormality in amylase or lipase), with no patient exhibiting clinical symptoms of severe pancreatitis [11]. In this study, we retrospectively investigated the symptoms and laboratory findings related to the GI system in a single center in Athens, Greece and assessed the role of these parameters in relation to patient survival and disease severity.

\section{Patients and methods}

We retrospectively studied 61 adult ( $>18$ years) patients with SARS-CoV-2 pneumonia admitted to the Army Share Fund Hospital (NIMTS) in Athens, Greece, between April 6th and May 6th, 2020. Since our hospital is a referral center for COVID-19 patients, the SARS-CoV-2 pneumonia diagnosis was established before these patients were transported from other healthcare facilities or from their residences.

The study was approved by the Institutional Review Board of our Hospital, as it was found to be consistent with the Helsinki declaration.

All cases had a history of exposure and clinical manifestations including fever and/or respiratory symptoms. The disease was confirmed by detecting SARS-CoV-2 nucleic acid in throat and nasal swab samples using the reverse transcription polymerase chain reaction (RT-PCR) assay method. Patients were considered to have a confirmed infection if the initial test result was positive, or if it was negative but repeat testing was positive.

Serious illness (upon admission) was defined by at least one of the following criteria: (i) respiratory rate $\geq 30 / \mathrm{min}$; (ii) pulse oximeter oxygen saturation $\left(\mathrm{SpO}_{2}\right) \leq 93 \%$ at rest; (iii) ratio of partial pressure of arterial oxygen $\left(\mathrm{PaO}_{2}\right)$ to fraction of inspired oxygen $\left(\mathrm{FiO}_{2}\right) \leq 300 \mathrm{mmHg}(1 \mathrm{mmHg}=0.133 \mathrm{kPa})$; and (iv) presence of hypotension or any organ failure [12].

During hospitalization, and after being afebrile for at least 3 days, each patient had a swab virus test performed every 3 days.

\section{Data collection}

The Acute Respiratory Infection Clinical Characterization Data Tool, developed by the International Severe Acute Respiratory and emerging Infection Consortium (ISARIC) and the World Health Organization (WHO), was used by 2 physicians (PT and AA) to collect relevant demographics, medical history, exposure history, comorbidities, symptoms, signs, laboratory findings, treatment (antiviral, antibiotic and corticosteroid therapies, and respiratory support) and outcome data; the patients' electronic medical records were used for data extraction.

\section{Therapeutic strategies}

All patients received upon admission and for 10 days: hydroxychloroquine $400 \mathrm{mg}$ b.i.d. (reduced to $200 \mathrm{mg}$ b.i.d. from the second day), azithromycin $500 \mathrm{mg}$ q.d. (reduced to 250 mg q.d. from the second day) and ceftriaxone 2 g q.d. Patients with chronic renal failure received vancomycin $500 \mathrm{mg}$ every other day during hemodialysis. Antibiotics use was adapted as necessary. All patients received upon admission low-dose lowmolecular-weight heparin according to their body weight.

Tocilizumab, a humanized anti-interleukin-6 receptor antibody (Ro-Actemra ${ }^{\oplus}$, Roche Holding AG, Basel) was administered to all COVID-19 patients with elevated C-reactive protein (CRP) levels who required supportive care in the intensive care unit (ICU). Tocilizumab was reconstituted with $100 \mathrm{~mL} 0.9 \%$ sodium chloride solution and administered by intravenous infusion over $60 \mathrm{~min}$. Patients with disseminated neoplasia, age $>80$ years, or multiple comorbidities with life expectancy less than a year were excluded.

All patients rested in bed and received nutritional support. Their vital signs and pulse oxygen saturation were monitored with appropriate oxygen therapy provided over time. Water and electrolyte balance was maintained in all patients.

\section{Laboratory examination}

On admission, all patients had a comprehensive laboratory panel test performed, including blood cytology, biochemistry and a chest computed tomography (CT) scan. Laboratory tests were repeated as appropriate. Peripheral leukocyte count, lymphocyte absolute value, CRP (0-0.5 mg/dL), prothrombin time and international normalized ratio, serum albumin $(\mathrm{g} / \mathrm{dL})$, liver function enzymes including alanine aminotransferase (ALT, 5-40 U/L), aspartate aminotransferase (AST, 5-40 U/L), lactate dehydrogenase (LDH, 100-220 U/L), $\gamma$-glutamyl transferase (GGT, 10-50 U/L), alkaline phosphatase (ALP, 40-140 U/L), and total bilirubin (TB, $0.2-1.1 \mathrm{mg} / \mathrm{dL}$ ) were routinely measured using standard methods. We also measured amylase (25-125 $\mathrm{U} / \mathrm{L})$, creatine phosphokinase (CPK, 24-170), troponin (0.7 ng/ $\mathrm{mL})$ and $\mathrm{D}$-dimer levels $(<0.5 \mathrm{ng} / \mathrm{mL})$. We defined abnormal liver damage as any parameter more than the upper limit of normal: ALT (40 U/L), AST (40 U/L), ALP (140 U/L), GGT (50 $\mathrm{U} / \mathrm{L}), \mathrm{TB}(1.1 \mathrm{mg} / \mathrm{dL}), \mathrm{LDH}(220 \mathrm{U} / \mathrm{L})$. Interleukin 6 (IL-6) and procalcitonin were not routinely assessed in our patient cohort.

\section{Patient discharge}

Patients were cleared for discharge if they had: i) clinical remission of respiratory symptoms; ii) radiographic evidence 
of improvement via a chest CT scan; iii) no fever for at least 3 days; and iv) 2 nasal-swab samples negative for SARS-CoV-2 RNA (obtained at least $24 \mathrm{~h}$ apart).

\section{Statistical analysis}

The statistical analysis was used to extract descriptive characteristics, reported as percentages for the categorical data and as median and interquartile range (IQR) for continuous data (such as age and laboratory results). Additionally, we assessed the role of various parameters in patient survival, as well as disease severity (GI symptoms, GI-related biochemical parameters, and inflammation markers). Both univariate and multivariate analyses were performed; the latter was performed in order to take into account the various confounders. Univariate analysis was performed via the chi-square method for the categorical data and by non-parametric tests for the arithmetic data. Specifically, we applied the Kruskal-Wallis test, since normality could not be confirmed by the Kolmogorov-Smirnov test. Variables with a P-value $<0.05$ in the univariate analysis were included in a logistic regression analysis using the backward stepwise elimination method. Two multivariate models were considered, the first related to patient's survival and the second for disease severity. Only parameters measured at patient admission were entered into these models and under the assumption that they were already statistically significant during the univariate analysis. Parameters, such as age, already known to have an important effect on both survival and disease severity were excluded from the multivariate analysis, since the aim was to investigate GI system-related parameters, and moreover because they were expected to dominate the analysis. The results of the multivariate analysis are presented in terms of the odds ratio (OR) for each variable that eventually remained in the model and cumulatively by the area under the curve (AUC) of the receiver operating characteristic (ROC) analysis. All tests were performed using the SAS 9.4 statistical package and the statistical significance for the complete study was set to $<0.05$.

\section{Results}

Sixty-one cases admitted with COVID-19 were assessed in the study period. The baseline characteristics of the study population, demographics, medical history and medication are shown in Table 1 . Only 8 (13.1\%) patients had no underlying comorbidity, while $15(24.6 \%)$ presented with $1,16(26.2 \%)$ with $2,16(26.2 \%)$ with 3 , and $6(9.8 \%)$ with $\geq 4$ comorbidities. Thirty-seven (60.7\%) patients had severe disease. The laboratory results on admission are summarized in Table 2.

Table 1 Baseline characteristics of the study population, demographics, medical history and previous medications

\begin{tabular}{|c|c|c|c|}
\hline Characteristics & $\mathrm{N}(\%)$ & Characteristics & $\mathbf{N}(\%)$ \\
\hline Age (in years) & 70 (55-83) median (IQR) & Severe infection before COVID-19 co-infection & $12(19.7 \%)$ \\
\hline Sex (male) & $34(55.7 \%)$ & Nephrolithiasis & $4(6.6 \%)$ \\
\hline Active smoking & $2(3.3 \%)$ & Liver cirrhosis & $1(1.6 \%)$ \\
\hline Rheumatic disease & $3(4.9 \%)$ & Beta thalassemia & $3(4.9 \%)$ \\
\hline Hypertension & $35(57.4 \%)$ & Depression & $2(3.3 \%)$ \\
\hline Ischemic heart disease & $13(21.3 \%)$ & Hyperlipidemia & $16(26.2 \%)$ \\
\hline Myocardial infarction & $7(11.5 \%)$ & Organic psychosyndrome & $5(8.2 \%)$ \\
\hline Atrial fibrillation & $10(16.4 \%)$ & Pancreatitis (chronic) & $1(1.6 \%)$ \\
\hline Peripheral vascular disease & $11(18 \%)$ & Autoimmune disease & $2(3.3 \%)$ \\
\hline Chronic renal failure & $26(42.6 \%)$ & Previous medications & \\
\hline Asthma/COPD & $8(13.1 \%)$ & Corticosteroids & $5(8.2 \%)$ \\
\hline Stroke & $5(8.2 \%)$ & Biologic agents & $1(1.6 \%)$ \\
\hline Dementia & $9(14.8 \%)$ & PPIs & $16(26.2 \%)$ \\
\hline Epilepsy & $3(4.9 \%)$ & Antihypertensives & $11(18 \%)$ \\
\hline Parkinson's disease & $1(1.6 \%)$ & Diuretics & $9(14.8 \%)$ \\
\hline Active neoplasia & $12(19.7 \%)$ & Beta blockers & $17(27.9 \%)$ \\
\hline Metastatic disease & $4(6.6 \%)$ & Chemotherapeutics & $4(6.6 \%)$ \\
\hline Thyroid disease & $9(14.8 \%)$ & Anticoagulants/antiplatelets & $5(8.2 \%)$ \\
\hline DM & $27(44.3 \%)$ & NSAIDs & $10(16.4 \%)$ \\
\hline
\end{tabular}

COPD, chronic obstructive pulmonary disease; DM, diabetes mellitus; PPIs, proton pump inhibitors; NSAIDs, non-steroidal anti-inflammatory drugs; $N$, number of positive cases, IQR, interquartile range 
At the end of our study, 45 (73.8\%) patients survived, 25 of whom had been discharged from our hospital, whereas 16 patients $(26.2 \%)$ passed away. Seven patients (43.8\%) died of cardiac arrest in the setting of severe lung disease, 8 (50\%) from severe deterioration of their respiratory function, while one $(6.2 \%)$ died from functional deterioration of an already cirrhotic liver and despite SARS-CoV-2 RNA clearance in throat and nasal swab samples.

\section{GI symptoms and patient survival}

Diarrhea was reported in 11 (24\%) of the 45 surviving patients, while no deceased patient had diarrhea $(\mathrm{P}=0.0289)$. Nausea and vomiting were present in 3 (7\%) of the 45 surviving patients and in only 1 (6\%) of the 16 deceased (OR 0.9, 95\% confidence interval [CI] 0.1-9.7, $\mathrm{P}=0.9539)$. Only 1 of the 45 surviving patients (2\%) and $1(6 \%)$ amongst the 16 deceased reported abdominal pain (OR 2.9, 95\%CI 1.7-49.9, $\mathrm{P}=0.4371$ ).

Table 2 Laboratory results on admission

\begin{tabular}{|c|c|}
\hline Biomarker & Median (IQR) \\
\hline $\mathrm{CRP}(\mathrm{mg} / \mathrm{dL})$ & $4.24(1.4-8.39)$ \\
\hline D-dimers $(\mu \mathrm{g} / \mathrm{mL})$ & $1(0.5-2.5)$ \\
\hline Neutrophil cell count $(/ \mu \mathrm{L})$ & $3300(2400-5700)$ \\
\hline Lymphocyte count $(/ \mu \mathrm{L})$ & $1200(900-1700)$ \\
\hline Hemoglobin (g/dL) & $11.8(10.1-13.4)$ \\
\hline Platelet count $(/ \mu \mathrm{L})$ & $212000(155000-317000)$ \\
\hline Ferritin (ng/mL) & $474(209-832)$ \\
\hline Blood glucose (mg/dL) & $96(81-119)$ \\
\hline Creatinine (mg/dL) & $1(0.8-4.8)$ \\
\hline CPK (U/L) & $74(44-146)$ \\
\hline $\mathrm{LDH}(\mathrm{U} / \mathrm{L})$ & $253(188-330)$ \\
\hline Total bilirubin (mg/dL) & $0.42(0.33-0.57)$ \\
\hline $\operatorname{AST}(\mathrm{U} / \mathrm{L})$ & $28(20-46)$ \\
\hline $\operatorname{ALT}(\mathrm{U} / \mathrm{L})$ & $22(15-39)$ \\
\hline GGT (U/L) & $28(19-59)$ \\
\hline ALP (U/L) & $55(43-74)$ \\
\hline Amylase (U/L) & $72(52-111)$ \\
\hline Albumin (g/dL) & $3(2.5-3.5)$ \\
\hline INR & $1.15(1.08-1.26)$ \\
\hline
\end{tabular}

$I Q R$, interquartile range; $C R P, C$-reactive protein; $A S T$, aspartate aminotransferase; ALT, alanine aminotransferase; INR, international normalized ratio; GGT, $\gamma$-glutamyl transferase: ALP, alkaline phosphatase; $C P K$, creatinine phosphokinase; $L D H$, lactate dehydrogenase

\section{GI symptoms and disease severity}

Diarrhea was recorded in 5 patients (21\%) of the 24 with mild disease and in 6 patients (16\%) of the 37 with severe disease (OR $0.7,95 \%$ CI $0.2-2.7, \mathrm{P}=0.6468)$. Nausea and vomiting were present in 2 patients (8\%) of the 24 with mild disease and in 2 patients (5\%) of the 37 with severe disease (OR 0.6, 95\%CI 0.1-4.8, $\mathrm{P}=0.6518$ ). No patient with mild disease reported abdominal pain, in contrast with 2 patients $(5 \%)$ of the 37 with severe disease $(\mathrm{P}=0.2468)$.

\section{Univariate analysis}

The univariate analysis of the relation between GI laboratory results and patients' survival is shown in Table 3. The univariate analysis of the relation between GI laboratory results and disease severity is shown in Table 4.

\section{Multivariate analysis}

Role of GI-related parameters in patient survival

The multivariate analysis of patient survival was based solely on the statistically important factors identified in the univariate analysis and incorporated GI symptoms, GI-related biochemical parameters, and inflammation markers. Two factors were eventually significant for survival: AST (OR 1.029, 95\%CI 1.007-1.05, $\mathrm{P}=0.0088$ ) and albumin levels (OR 0.219, 95\%CI $0.066-0.723, \mathrm{P}=0.0127)$, measured upon patient admission.

The characteristic curves showing the predicted probabilities for patient death as AST levels increase and albumin levels decrease are depicted in Fig. 1A,B. Notably, the AUC of the ROC curve related to the multivariate model based on AST and albumin was $85.6 \%$.

\section{Role of Gl-related parameters in disease severity}

The multivariate analysis of disease severity indicated that, ultimately, of the parameters that emerged as significant from the univariate analysis, only albumin measured upon patient admission remained as an independent variable. The OR for albumin was 0.025 (95\%CI $0.004-0.161, \mathrm{P}=0.0001)$. The AUC for the relevant ROC curve was $89.0 \%$. The predicted probability for severe disease in relation to albumin levels is shown in Fig. 2.

\section{Discussion}

With numerous new COVID-19 patients being reported every day, our experience regarding their clinical presentation and possible therapeutic choices and prognostic factors is increasing. These patients may exhibit GI involvement, with diarrhea and/or vomiting/nausea being the most common symptoms $[9,12]$. Besides the clinical presentation, abnormal 
Table 3 Univariate analysis of patient survival and gastrointestinal-related laboratory results

\begin{tabular}{|c|c|c|c|}
\hline \multirow[t]{2}{*}{ Biomarkers } & Surviving patients $(\mathrm{N}=45)$ & Deceased patients $(\mathrm{N}=16)$ & \multirow[t]{2}{*}{ P-value } \\
\hline & Median (IQR) & Median (IQR) & \\
\hline Albumin $(\mathrm{g} / \mathrm{dL})$ & $3.3(2.9-3.7)$ & $2.45(2.35-3)$ & 0.0007 \\
\hline $\operatorname{ALP}(\mathrm{U} / \mathrm{L})$ & $55(43-74)$ & $55(44-73)$ & 0.8184 \\
\hline t-bil (mg/dL) & $0.43(0.33-0.58)$ & $0.41(0.355-0.475)$ & 0.7243 \\
\hline $\mathrm{CPK}(\mathrm{U} / \mathrm{L})$ & $66(43-117)$ & $149.5(52.5-374.5)$ & 0.0351 \\
\hline INR & $1.14(1.08-1.22)$ & $1.245(1.14-1.535)$ & 0.0335 \\
\hline AST (U/L) & $26(20-33)$ & $47.5(25.5-138.5)$ & 0.0093 \\
\hline $\operatorname{ALT}(\mathrm{U} / \mathrm{L})$ & $22(16-36)$ & 25 (13.5-109) & 0.7992 \\
\hline GGT (U/L) & $32(19-69)$ & $25(19-40)$ & 0.3539 \\
\hline Amylase (U/L) & $72(55-111)$ & $67.5(31-104)$ & 0.3891 \\
\hline $\mathrm{LDH}(\mathrm{U} / \mathrm{L})$ & $253(203-315)$ & $251.5(115-483.5)$ & 0.7430 \\
\hline $\mathrm{CRP}(\mathrm{mg} / \mathrm{dL})$ & $2.3(1.09-7.4)$ & $7.305(3.69-14.45)$ & 0.0085 \\
\hline D-dimers $(\mu \mathrm{g} / \mathrm{mL})$ & $0.7(0.5-1.7)$ & $2.4(1-2.8)$ & 0.0125 \\
\hline Ferritin $(\mathrm{ng} / \mathrm{mL})$ & $357(151-821)$ & $691(341-1437.5)$ & 0.0725 \\
\hline Lymphocytes $(/ \mu \mathrm{L})$ & $1300(1000-1700)$ & $1000(750-1300)$ & 0.2402 \\
\hline PLTs $(/ \mu \mathrm{L})$ & $214000(162000-317000)$ & $197500(123000-319500)$ & 0.4606 \\
\hline
\end{tabular}

ALP, alkaline phosphatase; CPK, creatinine phosphokinase; INR, international normalized ratio; $t$-bil, total bilirubin; AST, aspartate aminotransferase; $A L T$, alanine aminotransferase; GGT, $\gamma$-glutamyl transferase; LDH, lactate dehydrogenase; CRP, C-reactive protein; PLTs, platelets N, number of patients in each group; $I Q R$, interquartile range

Table 4 Univariate analysis of disease severity and gastrointestinal-related laboratory results

\begin{tabular}{|c|c|c|c|}
\hline \multirow[t]{2}{*}{ Biomarkers } & Mild disease $(\mathrm{N}=24)$ & Severe disease $(\mathrm{N}=37)$ & \multirow[t]{2}{*}{$\mathrm{P}$-value } \\
\hline & Median (IQR) & Median (IQR) & \\
\hline Albumin (g/dL) & $3.5(3.3-3.9)$ & $2.8(2.4-3)$ & $<0.0001$ \\
\hline $\operatorname{ALP}(\mathrm{U} / \mathrm{L})$ & $53.5(40.5-75.5)$ & $55(43-70)$ & 0.6846 \\
\hline $\mathrm{t}$-bil (mg/dL) & $0.46(0.335-0.655)$ & $0.41(0.33-0.49)$ & 0.3636 \\
\hline CPK (U/L) & $68(46-123)$ & $87(43-153)$ & 0.8420 \\
\hline INR & $1.125(1.08-1.22)$ & $1.16(1.11-1.28)$ & 0.1692 \\
\hline $\operatorname{AST}(\mathrm{U} / \mathrm{L})$ & $22.5(20-30)$ & $33(23-54)$ & 0.0400 \\
\hline $\operatorname{ALT}(\mathrm{U} / \mathrm{L})$ & $23.5(15.5-40)$ & $22(15-38)$ & 0.9529 \\
\hline GGT (U/L) & $23.5(18-64)$ & $31(24-50)$ & 0.3332 \\
\hline Amylase (U/L) & $67.5(51-100)$ & $76(54-111)$ & 0.5596 \\
\hline $\mathrm{LDH}(\mathrm{U} / \mathrm{L})$ & $215.5(183-261)$ & $280(237-384)$ & 0.0102 \\
\hline $\mathrm{CRP}(\mathrm{mg} / \mathrm{dL})$ & $2.145(0.875-6.515)$ & $5.38(2.16-12)$ & 0.0171 \\
\hline D-dimers $(\mu \mathrm{g} / \mathrm{mL})$ & $0.65(0.4-1.25)$ & $1.4(0.7-2.8)$ & 0.0150 \\
\hline Ferritin (ng/mL) & $312.5(146-748)$ & $543(283-1309)$ & 0.0681 \\
\hline Lymphocytes $(/ \mu \mathrm{L})$ & $1250(950-1600)$ & $1200(800-1800)$ & 0.7060 \\
\hline PLTs $(/ \mu \mathrm{L})$ & $196000(158000-309500)$ & $214000(154000-317000)$ & 0.6632 \\
\hline
\end{tabular}

ALP, alkaline phosphatase; CPK, creatinine phosphokinase; INR, international normalized ratio; $t$-bil, total bilirubin; AST, aspartate aminotransferase; ALT, alanine aminotransferase; GGT, $\gamma$-glutamyl transferase; $L D H$, lactate dehydrogenase; CRP, C-reactive protein; PLTs, platelets N, number of patients in each group; $I Q R$, interquartile range

laboratory findings relating to the GI system (enteric, hepatic, biliary, and pancreatic) can also be expected. The pathophysiology behind these findings is complicated and not completely understood. Angiotensin-converting enzyme 2-mediated direct viral infection of enterocytes, hepatocytes, cholangiocytes and pancreatic islet cells, direct injury of the GI system due to an inflammatory response (especially in the event of multiple organ dysfunction syndrome in critical 


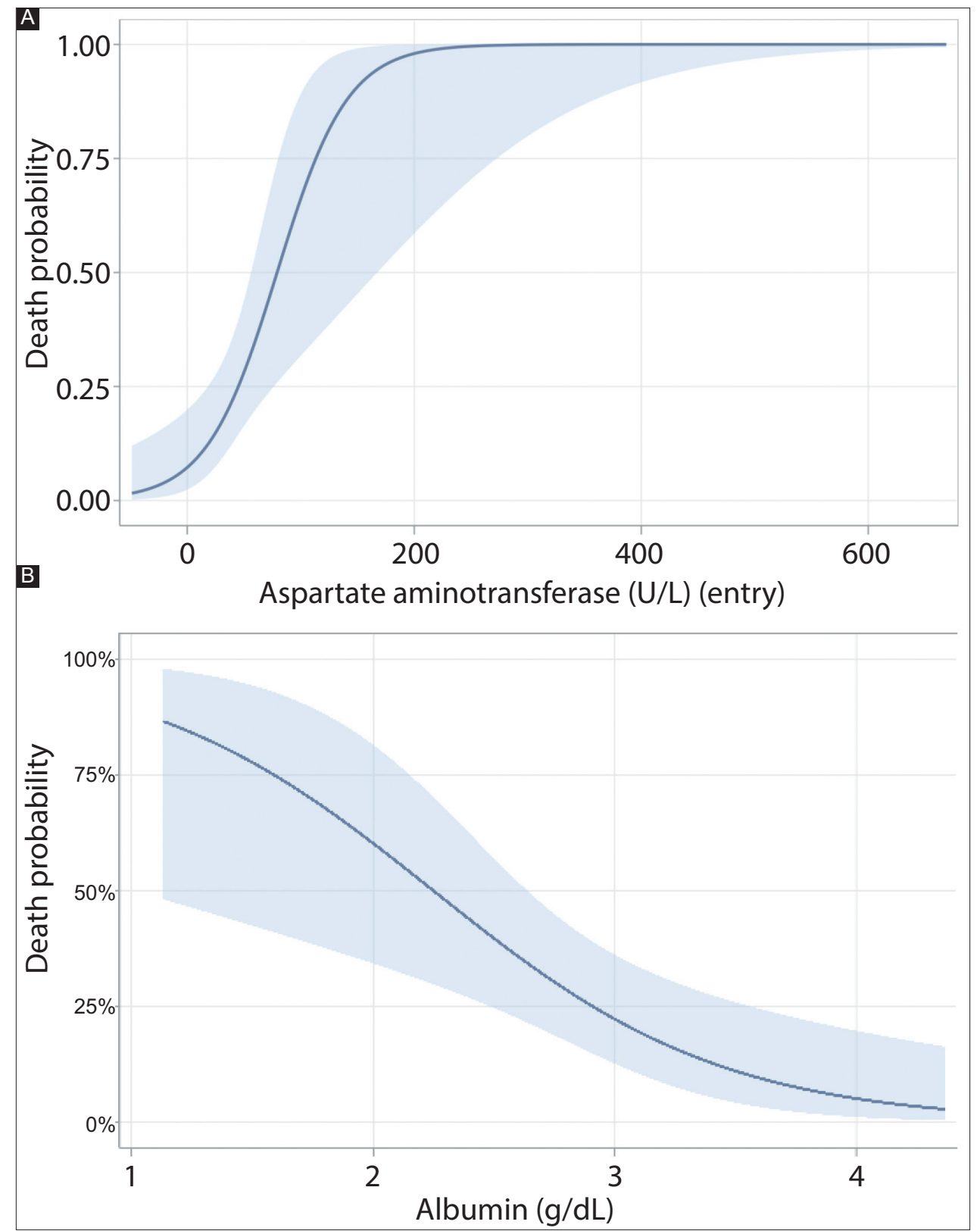

Figure 1 The predicted probabilities for patient death in relation to aspartate aminotransferase (A) and albumin (B) levels on admission Continuous lines indicate the estimated probability from the multivariate model, while the shaded area represents the 95\% confidence intervals

COVID-19 cases), immune-mediated injury, or even drug toxicity, are all implicated $[11,13,14]$.

We tried to implement our clinical experience from being a COVID-19 referral hospital so as to retrospectively report, for the first time in Greece, the GI involvement in COVID-19 patients. Regarding both survival and disease severity, diarrhea was the most common finding, followed by nausea/vomiting and abdominal pain; this was as expected and in accordance with previous reports and meta-analyses [12,14]. Notably, no statistical significance was noted for all the aforementioned findings; this is in accordance with a recent meta-analysis in a Chinese population, where the prevalence of GI symptoms had no significance in discriminating between severe and non-severe disease and had no prognostic role [15]. However, another meta-analysis reported contrary findings, where patients with severe COVID-19 had higher rates of GI symptoms and patients with GI involvement revealed a higher prevalence of complications [16].

These contradictory data can be expected when researching a previously unexplored field in a newly emerged pandemic disease. The number of patients in our study was relatively small and, as the current literature suggests, there are geographical differences in COVID-19 populations regarding disease presentation and prognosis [9]. A multicenter cohort study from the United States reported that, overall, $61.3 \%$ of patients reported at least one GI symptom on presentation, most commonly anorexia (34.8\%), diarrhea $(33.7 \%)$, or nausea $(26.4 \%)$, and that GI symptoms were the predominant presenting complaint among $20.3 \%$ of 


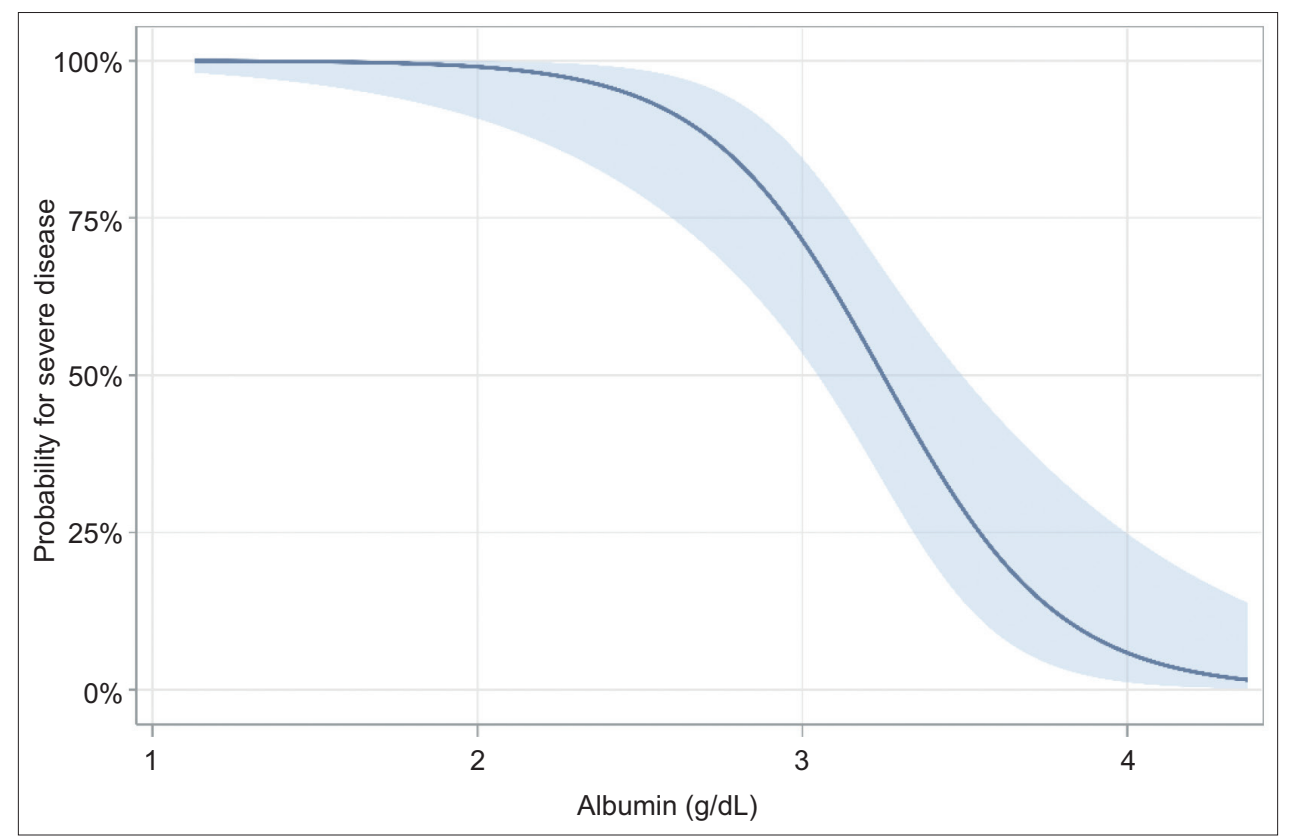

Figure 2 The predicted probability for severe disease in relation to albumin levels on admission

The continuous line indicates estimated probability from the multivariate model, while the shaded area represents the 95\% confidence interval

patients and the initial presenting symptoms of COVID-19 among $14.2 \%$ of individuals [17]. Wan et al retrospectively analyzed data from 232 patients positive for SARS-CoV-2 RNA admitted to 14 hospitals in different geographic locations in China. The authors reported more patients with diarrhea exhibiting severe symptoms of pneumonia compared to those without diarrhea (adjusted for age, sex and comorbidities) and a greater proportion of patients with diarrhea also requiring ventilator support and admission to the ICU. Moreover, a greater proportion of patients were admitted to hospital with diarrhea as the outbreak progressed, suggesting a heightened awareness of the GI symptoms of COVID-19 among clinicians and/or possible SARS-CoV-2 accumulative mutations affecting the virus's tropism for the intestines [18].

Regarding the role of GI-related laboratory findings, while the univariate analysis initially revealed that numerous biochemical parameters measured upon patient admission had an impact on patient survival and disease severity, the multivariate analysis revealed that increased serum AST levels and decreased serum albumin levels were associated with poorer patient survival (OR 1.029, 95\%CI 1.007-1.05, $\mathrm{P}=0.0088$; and $\mathrm{OR} 0.219,95 \% \mathrm{CI} 0.066-0.723, \mathrm{P}=0.0127$, respectively). As far as disease severity was concerned, only a low serum albumin level on patient admission was correlated with more severe disease (OR 0.025, 95\%CI 0.004-0.161, $\mathrm{P}=0.0001$ ).

The AGA reports a pooled prevalence of elevated liver abnormalities for AST 15.0\% (95\%CI 13.6-16.5\%) and ALT $15.0 \%$ (95\%CI 13.6-16.4\%), rendering liver enzyme testing an important parameter that should be examined in all COVID-19 patients [9]. In a meta-analysis of 12 studies and a population of 1267 COVID-19 patients Mao et al reported that the pooled prevalence of abnormal liver function was 19\%, with patients with severe COVID-19 having higher rates of liver injury (OR 2.20, 95\%CI 1.60-3.02, $\mathrm{P}=0.00001$ ) [16]. Huang et al related AST elevation to the probability of COVID-19 patients entering the ICU [19]. Moreover, a large cohort of 1099 patients reported more patients with severe disease and abnormal aminotransferase levels, possible as a result of multiorgan dysfunction [1]. Lower serum albumin was also reported in a retrospective, multicenter cohort study from China in patients with laboratory confirmed COVID-19. The median values of albumin upon hospital admission for nonsurvivors vs. survivors were $29.1 \mathrm{~g} / \mathrm{L}$ and $33.6 \mathrm{~g} / \mathrm{L}$, respectively $(\mathrm{P}<0.0001)$ [20]. A significant correlation was found between albumin level and inflammatory indicators. Inflammation has been shown to cause the escape of serum albumin into interstitial space by increased capillary permeability, eventually leading to an increased volume of albumin distribution [21,22]. A meta-analysis has shown that about $80.4 \%$ of COVID-19 patients with abnormal liver function had hypoalbuminemia, associated with a poor prognosis and outcome [23]. Moreover, hypoalbuminemia has also been associated with the severity of acute respiratory distress syndrome [24] and acute kidney injury [25].

Our study has some limitations that should be noted. First, our study population came from a single centre and included numerous patients with chronic renal failure on dialysis; the effect of other comorbidities (i.e., cancer) was not sought. Second, the outcome for many of our patients was unknown at the cutoff time set for data collection. Third, data generation was clinically driven and not systematic. Fourth, we did not assess for treatment-related GI and hepatic adverse effects.

In conclusion, both the United States Centers for Disease Control and Prevention (CDC) and the AGA advocate that, in outpatients with new-onset GI symptoms (e.g., nausea, vomiting, abdominal pain, diarrhea), COVID-19 testing should be considered in a high COVID-19 prevalence setting (priorities for COVID-19 testing) $[9,26]$. The vast majority of studies of GI involvement in 
COVID-19 patients are retrospective, and epidemiological studies from numerous countries as well as studies concerning COVID-19 prognosis are eagerly awaited. As prospective studies begin to emerge [27] and systematic review and meta-analyses data are published $[28,29]$, clinicians will have more robust research data that will offer the opportunity to: diagnose COVID-19 patients earlier; use prognostication in their everyday clinical practice; and identify patients in need of more intensive treatment.

\section{Summary Box}

\section{What is already known:}

- Much attention has been paid to the study and reporting of gastrointestinal symptoms in COVID-19 patients

- An increasing number of COVID-19 patients have been noted to experience hepatic and pancreatic injury

\section{What the new findings are:}

- Increased aspartate aminotransferase serum levels and decreased albumin levels at patient admission may be associated with poorer patient survival

- Decreased albumin levels at patient admission may be associated with more severe disease

\section{References}

1. Guan WJ, Ni ZY, Hu Y, et al; China Medical Treatment Expert Group for COVID-19. Clinical characteristics of coronavirus disease 2019 in China. N Engl J Med 2020;382:1708-1720.

2. Holshue ML, DeBolt C, Lindquist $S$, et al; Washington State 2019-nCoV Case Investigation Team. First case of 2019 novel coronavirus in the United States. N Engl J Med 2020;382:929-936.

3. WHO Coronavirus disease (COVID-19) dashboard. Available from: https://covid19.who.int/ [Accessed 15 June 2020].

4. John Hopkins Coronavirus Resource Center. Available from: https://coronavirus.jhu.edu/map.html [Accessed 15 June 2020].

5. Wei XS, Wang X, Niu YR, et al. Diarrhea is associated with prolonged symptoms and viral carriage in COVID-19. Clin Gastroenterol Hepatol 2020 Apr 18 [Online ahead of print]. doi: 10.1016/j.cgh.2020.04.030

6. Yang L, Tu L. Implications of gastrointestinal manifestations of COVID-19. Lancet Gastroenterol Hepatol 2020 May 12 [Online ahead of print]. doi: 10.1016/S2468-1253(20)30132-1

7. Grassia R, Testa S, Pan A, Conti CB. SARS-CoV-2 and gastrointestinal tract: the dark side of the pandemic. Dig Liver Dis 2020 Apr 29 [Online ahead of print]. doi: 10.1016/j.dld.2020.04.028

8. Nobel YR, Phipps M, Zucker J, et al. Gastrointestinal symptoms and COVID-19: case-control study from the United States. Gastroenterology 2020 Apr 12 [Online ahead of print]. doi: 10.1053/j.gastro.2020.04.017

9. Sultan S, Altayar O, Siddique SM, et al. AGA Institute rapid review of the GI and liver manifestations of COVID-19, meta-analysis of international data, and recommendations for the consultative management of patients with COVID-19. Gastroenterology 2020
May 11 [Online ahead of print]. doi: 10.1053/j.gastro.2020.05.001

10. Fan Z, Chen L, Li J, et al. Clinical features of COVID-19-related liver functional abnormality. Clin Gastroenterol Hepatol 2020;18:1561-1566.

11. Wang F, Wang H, Fan J, Zhang Y, Wang H, Zhao Q. Pancreatic injury patterns in patients with COVID-19 pneumonia. Gastroenterology 2020 Apr 1. [Online ahead of print]. doi: 10.1053/j.gastro.2020.03.055

12. Mo P, Xing Y, Xiao Y, Deng L, et al. Clinical characteristics of refractory COVID-19. Clin Infect Dis 2020 Mar 16 [Online ahead of print]. doi: 10.1093/cid/ciaa270

13. Liu Y, Xiang L, Deng K. Focusing on gastrointestinal symptoms in COVID-19 is far from enough. Gastroenterology 2020 May 18. [Online ahead of print]. doi: 10.1053/j.gastro.2020.05.043

14. Patel KP, Patel PA, Vunnam RR, et al. Gastrointestinal, hepatobiliary, and pancreatic manifestations of COVID-19. J Clin Virol 2020;128:104386.

15. Wang H, Qiu P, Liu J, Wang F, Zhao Q. The liver injury and gastrointestinal symptoms in patients with coronavirus disease 19: a systematic review and meta-analysis. Clin Res Hepatol Gastroenterol 2020 May 12 [Online ahead of print]. doi: 10.1016/j.clinre.2020.04.012

16. Mao R, Qiu Y, He J-S, et al. Manifestations and prognosis of gastrointestinal and liver involvement in patients with COVID-19: a systematic review and meta-analysis. Lancet Gastroenterol Hepatol 2020 May 12 [Online ahead of print]. doi: 10.1016/S2468-1253(20)30126-6

17. Redd WD, Zhou JC, Hathorn KE, et al. Prevalence and characteristics of gastrointestinal symptoms in patients with SARS-CoV-2 infection in the United States: a multicenter cohort study. Gastroenterology 2020 Apr 22 [Online ahead of print]. doi: 10.1053/j.gastro.2020.04.045

18. Wan Y, Li J, Shen L, et al. Enteric involvement in hospitalised patients with COVID-19 outside Wuhan. Lancet Gastroenterol Hepatol 2020;5:534-535.

19. Huang C, Wang Y, Li X, et al. Clinical features of patients infected with 2019 novel coronavirus in Wuhan, China. Lancet 2020;395:497-506.

20. Zhou F, Yu T, Du R, et al. Clinical course and risk factors for mortality of adult inpatients with COVID-19 in Wuhan, China: a retrospective cohort study. Lancet 2020;395:1054-1062.

21. Qin C, Zhou L, Hu Z, et al. Dysregulation of immune response in patients with COVID-19 in Wuhan, China. Clin Infect Dis 2020 Mar 12 [Online ahead of print]. doi: 10.1093/cid/ciaa248

22. Soeters PB, Wolfe RR, Shenkin A. Hypoalbuminemia: pathogenesis and clinical significance. JPEN J Parenter Enteral Nutr 2019;43:181-193.

23. Wu Y, Li H, Xu X, Zheng K, Qi X, Guo X. Clinical features and outcome of treatment for novel coronavirus pneumonia: a metaanalysis. Zhonghua Gan Zang Bing Za Zhi 2020;28:240-246.

24. Hoeboer SH, Oudemans-van Straaten HM, Groeneveld AB. Albumin rather than C-reactive protein may be valuable in predicting and monitoring the severity and course of acute respiratory distress syndrome in critically ill patients with or at risk for the syndrome after new onset fever. BMC Pulm Med 2015;15:22.

25. Wang B, Li D, Cheng B, Ying B, Gong Y. The neutrophil percentageto-albumin ratio is associated with all-cause mortality in critically ill patients with acute kidney injury. Biomed Res Int 2020;2020:5687672.

26. Evaluating and testing persons for coronavirus disease 2019 (COVID-19). Available from: https://www.cdc.gov/coronavirus/2019ncov/hcp/clinical-criteria.html [Accessed 15 June 2020].

27. Chen A, Agarwal A, Ravindran N, To C, Zhang T, Thuluvath PJ. Are gastrointestinal symptoms specific for COVID-19 infection? A prospective case-control study from the United States. Gastroenterology 2020 May 15 [Online ahead of print]. doi: 10.1053/j.gastro.2020.05.036

28. Piciucchi M, Sbrozzi-Vanni A, Rossi A, et al. Preliminary considerations regarding the risk of COVID-19 and disease severity in chronic gastrointestinal conditions. Ann Gastroenterol 2020;33:327-329.

29. Rokkas T. Gastrointestinal involvement in COVID-19: a systematic review and meta-analysis. Ann Gastroenterol 2020;33:355-365. 